\title{
Lunasin: A promising polypeptide for the prevention and treatment of cancer (Review)
}

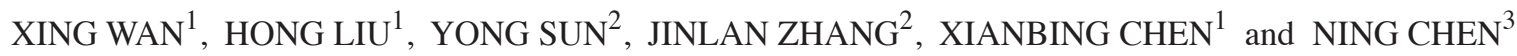 \\ ${ }^{1}$ School of Medicine, Hubei University for Nationalities, Enshi, Hubei 445000; \\ ${ }^{2}$ China Meat Research Centre, Beijing 100068; ${ }^{3}$ Hubei Key Laboratory of Sport Training and Monitoring, \\ College of Health Science, Wuhan Sports University, Wuhan, Hubei 430079, P.R. China
}

Received October 7, 2015; Accepted January 10, 2017

DOI: $10.3892 / \mathrm{ol} .2017 .6017$

\begin{abstract}
Strategies for the treatment of cancer remain unsatisfactory due to the poor understanding of the complicated underlying molecular mechanisms of carcinogenesis. A number of types of cancer exhibit a marked association with dietary habits and lifestyles. Therefore, the modulation of dietary habits or lifestyles may be an effective strategy for preventing the formation and progression of cancer. Proteins and polypeptides from soybean have been developed as healthcare products due to their marked activity in inhibiting the progression of cancer at various stages. Lunasin, containing 43 amino acid residues, is one such example of a soybean-derived polypeptide that has been demonstrated to exhibit marked anti-cancer activity. In the present review, studies of the underlying molecular mechanisms and potential advantages of lunasin in the prevention and treatment of cancer have been examined, to provide a theoretical reference for the development of natural product-based agents or healthcare products for the prevention and treatment of cancer.
\end{abstract}

\section{Contents}

1. Introduction

2. Structure of lunasin

3. Characterization of lunasin

4. Molecular mechanisms and advantages of lunasin, in the prevention and treatment of cancer

5. Conclusions

Correspondence to: Professor Ning Chen, Hubei Key Laboratory of Sport Training and Monitoring, College of Health Science, Wuhan Sports University, 461 Luoyu Road, Wuhan, Hubei 430079, P.R. China

E-mail: nchen510@gmail.com

Professor Xianbing Chen, School of Medicine, Hubei University for Nationalities, 39 Xueyuan Road, Enshi, Hubei 445000, P.R. China

E-mail: chenxianbing7612@163.com

Key words: lunasin, polypeptide, cancer prevention, dietary habit, healthcare product, anti-cancer mechanism

\section{Introduction}

Cancer is well-known to be one of the leading causes of mortality worldwide (1). However, the therapy of various types of cancer remains unsatisfactory due to the poor understanding of the complicated underlying molecular mechanisms of carcinogenesis. A number of epidemiological studies have demonstrated that a number of types of cancer exhibit a marked association with dietary habits and lifestyles (2-4). A statistical study has demonstrated that 3.07 million individuals in China were diagnosed with cancer in 2012, which accounts for $1 / 5$ of the total number of patients with cancer worldwide (5). Furthermore, $\sim 2.21$ million patients succumbed to cancer in China, which is $\sim 25 \%$ of the total mortality worldwide due to cancer (5). Epidemiological evidence has demonstrated that cancer in $35 \%$ of patients exhibits a marked association with lifestyle, particularly diet (2). Therefore, the adjustment of dietary habits and lifestyles may be an effective strategy to prevent carcinogenesis. Cancer cell- and tumor-bearing animal models have demonstrated that the consumption of foods containing natural compounds with anti-cancer activity is able to markedly reduce the risk of cancer, and increase the sensitivity of cancer cells to treatment (6). A number of phytochemicals, including resveratrol, quercetin and flavonoids, have been demonstrated to exhibit marked anti-cancer activity (7-9). Similarly, foodborne proteins and polypeptides have attracted attention due to their specific advantages as anti-cancer substances $(10,11)$. Compared with certain small-molecule drugs, polypeptides exhibit characteristics of increased affinity, marked ability of specific targeting and decreased toxicity; furthermore, polypeptides exhibit increased permeability in tissues compared with protein-based drugs (12). Therefore, polypeptides have been recognized as potential natural anti-cancer substances for inhibiting the development and progression of cancer at different stages (13).

Following extensive exploitation and utilization of natural products, soybean and other associated products have aroused interest from consumers due to their health-promoting benefits (14). Previous studies have demonstrated that increased soybean consumption in Asian populations is associated with decreased incidence 
of osteoporosis, cardiovascular disease and cancer (15-17). Furthermore, soybean products may reduce the risk of developing prostate and breast cancer $(18,19)$. Soybean was demonstrated to contain a variety of bioactive compounds, including protease inhibitors, lunasin, sitosterol, saponins and isoflavones, with significant anti-cancer activity $(20,21)$. Bowman-Birk protease inhibitor (BBI) and isoflavones have been investigated extensively $(18,20,22)$. Similarly, lunasin, isolated and extracted from soybean or other similar plants, has been reported as a polypeptide with a clear function to inhibit chemical-induced carcinogenesis (14). In the present review, studies of the underlying molecular mechanisms and corresponding advantages of lunasin for the prevention and treatment of cancer have been systematically examined to provide a theoretical reference, for the development of natural product-based agents or healthcare products, for the prevention and treatment of cancer.

\section{Structure of lunasin}

Lunasin, a polypeptide with molecular mass of $5.5 \mathrm{kDa}$, is able to enter mammalian cells within min and target nuclei within $18 \mathrm{~h}$ (23). Lunasin contains 43 amino acid residues, which comprise a fragment with an unknown function (residues 1-22), a helical structure (residues 23-31) that is similar to the conserved structure of chromatin-binding protein for binding to histones $\mathrm{H} 3 / \mathrm{H} 4$, a cell adhesion module of Arg-Gly-Asp (RGD) (residues 32-34) that functions as an extracellular matrix to promote the intracellular accessibility of lunasin and a critical fragment of nine aspartic acids at the C-terminus that is involved in anti-mitotic functions (23). The full sequence of lunasin is SKWQHQQDSCRKQLQGVNLT PCEKHIMEKIQGRDDDDDDDDDD and the fragments are depicted in Fig. 1 (14).

\section{Characterization of lunasin}

Lunasin has been identified in soybean, barley, wheat and rye, and has been subjected to corresponding comparative analysis $(24,25)$. However, due to the genotypic impact of different species, the lunasin content varies between species (26). The lunasin content in soybean, barley, wheat and rye was determined to be between 0.5 and 8.1 , between 0.013 and 0.099 , between 0.2 and 0.3 , and between 0.045 and $0.150 \mathrm{mg} / \mathrm{g}$ seed, respectively $(14,25,26)$. Therefore, soybean and wheat are recognized as the principal sources of lunasin (14).

The common extraction procedures for lunasin include crushing, defatting, extraction, dialysis, centrifugation and determination (14). This extraction method usually results in a crude protein containing lunasin, which requires further purification. Ion-exchange column chromatography has been used for the purification of lunasin extracted from soybean and barley in previous studies, and the principal material used in the ion-exchange column is bio-gel resin AG1-X4 with a particle size of between 100 and 200 mesh, and the volume of the ion-exchange column is $5.0 \times 50 \mathrm{~cm}$ with a filled height of $40 \mathrm{~cm}(27,28)$. In addition, gel electrophoresis, western blotting and mass spectrometry are also used for the qualitative analysis and quantitative determination of lunasin $(29,30)$.

\section{Molecular mechanisms and advantages of lunasin, in the prevention and treatment of cancer}

Advantages of lunasin in the prevention and treatment of cancer. An important characteristic of the ideal agents or health-promoting products for the prevention and treatment of cancer is their marked bioactivity in the inhibition of cancer cell generation and growth. Following oral administration of lunasin, it is absorbed into the bloodstream and reaches target tissues or organs in an active and stable state (31). Animal studies using rats fed on a soybean diet rich in lunasin demonstrated that the marked stability of lunasin enables it to reach target tissues and organs following administration, thus serving an important role in the prevention of cancer $(24,32)$. The bioactivity of lunasin has been investigated in humans, and results have demonstrated that $4.5 \%$ lunasin at full activity is able to enter the plasma of healthy volunteers in $30 \mathrm{~min}$ (33). In spite of the marked bioactivity of lunasin following administration, another study has demonstrated that $\mathrm{BBI}$ is required to protect lunasin from digestion (34). This protective role provides the favorable conditions required for lunasin to perform its functions. In addition, its marked stability following administration may be due to its specific secondary or tertiary structure, which remains unclear and requires further study. These studies provide the scientific basis for understanding the functions of lunasin in the prevention and treatment of cancer, and provide a rationale for the development of lunasin-based health-promoting products or drugs used in the treatment of cancer.

Molecular mechanisms of lunasin in the prevention and treatment of cancer. A previous study has demonstrated that lunasin is able to suppress the proliferation and migration of cancer cells caused by chemical carcinogens without any impact on wild-type cells (35). The potential underlying molecular mechanisms of lunasin in the prevention and treatment of cancer have been systematically investigated. Lunasin exhibits anti-mitotic effects, and may prevent the mutation caused by the chemical carcinogen 7,12-dimethylaminobenzaldehyde (DMAB) and viral oncogene early region $1 \mathrm{~A}(\mathrm{E} 1 \mathrm{~A})$ in mammals (36). When DMAB or methylcholanthrene is present alone, lunasin at between $10 \mathrm{nmol} / 1$ and $10 \mu \mathrm{mol} / 1$, was able to inhibit the mutation of cells by between 62 and $90 \%$ (36). In retinoblastoma $(\mathrm{Rb})$ and $\mathrm{DMAB}$-induced mouse embryonic fibroblasts, lunasin was able to inhibit the expression of oncogenes, the acetylation of histone $\mathrm{H} 3$ and the migration of tumor cells, and regulate the cell cycle to induce apoptosis, thereby inhibiting tumor formation $(36,37)$. In human breast cancer MDA-MB-231 cells, lunasin was able to reduce the expression of cyclin D1/D3, and down-regulate protein kinase $\mathrm{B} \alpha(\mathrm{PKB} \alpha)$ and activator protein 1 (AP-1), thereby inhibiting cell proliferation and inducing apoptosis $(38,39)$. In human leukemia L1210 cells, lunasin was able to reduce cell proliferation and induce apoptosis $(40,41)$. Similarly, in DMAB-induced mouse skin tumors, lunasin was able to decrease the number of tumors generated and delay the generation of tumors in skin $(42,43)$. Therefore, lunasin has been identified as a promising polypeptide for preventing tumor formation induced by chemical carcinogens. 


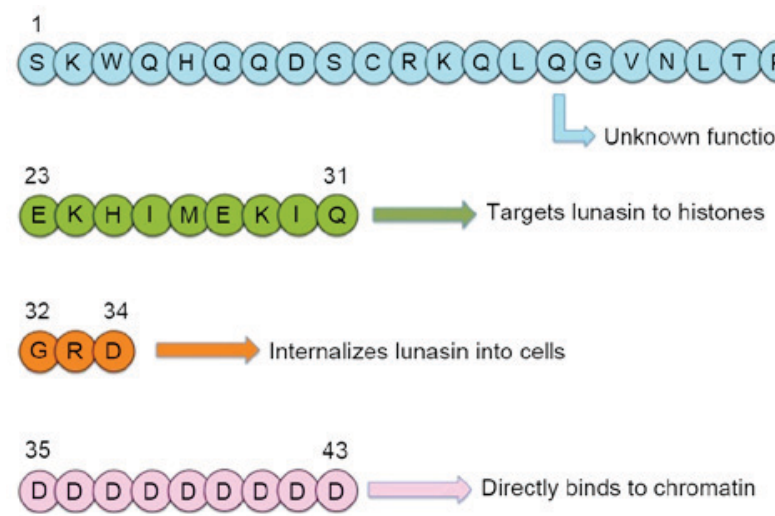

Figure 1. Sequence and structure of the fragments of lunasin. Figure previously published in (14)

In addition to the above-mentioned inhibition of cancer cell proliferation, epigenetic mechanisms have also been specifically analyzed during the application of lunasin. Acetylation is one of the most important modifications in cancer signaling pathways $(44,45)$. DNA damage and the disruption of chromatin transcription demonstrate a marked association with the occurrence of acetylation of specific lysine residues on histones (46). Under normal circumstances, histones $\mathrm{H} 3$ and $\mathrm{H} 4$ are in a deacetylated, or suppressed state. Therefore, the decreased activity of histone acetyltransferases (HATs), including lysine acetyltransferase 2A/2B and p300, is consistent with the decreased incidence of cancer, including colorectal and breast cancer (47-49). In previous studies, lunasin was demonstrated to competitively inhibit HATs, thereby inhibiting acetylation and regulating the cell cycle $(24,32)$. The underlying molecular mechanisms of the binding of lunasin to histones remain unclear; however, they may be associated with the helical structure formed by the amino acid residues at positions 23-31, and the specific spatial structure or orientation of lunasin (42). However, the inhibitory activity of histone acetylation was also markedly associated with the concentration of lunasin. Lunasin at a concentration of $10 \mathrm{nmol} / 1$ was able to decrease the acetylation of histones by between 20 and $25 \%$; by contrast, lunasin at a concentration $<1 \mu \mathrm{mol} / \mathrm{l}$ was able to decrease the acetylation of histones $\mathrm{H} 3$ and $\mathrm{H} 4$ by 80 and $74 \%$, respectively (50). Lunasin was able to act on cells at the stage of division or transformation, which is described by the E1A-Rb-histone deacetylase (HDAC) model (Fig. 2) (51). The tumor suppressor protein $\mathrm{Rb}$ interacted with eukaryotic initiation factor to supplement HDAC and maintain the deacetylated state of core histones (52). E1A resulted in the inactivation of $\mathrm{Rb}$, thus leading to the disassociation of the Rb-HDAC complex and the exposure of acetylated histones (52). Following acetylation of HATs, lunasin and HATs competitively deacetylate histones to terminate transcription, thus leading to apoptosis. Therefore, lunasin is involved in the destruction of acetylation-deacetylation kinetics of histones (32). Deacetylated histones are absent from wild-type cells, so lunasin was not able to disrupt the proliferation or cause the apoptosis of wild-type cells. While monitoring the cell cycle process in the presence of lunasin, lunasin was able to be activated to selectively kill tumor cells following the transformation of the cells from wild-type cells to tumor cells,

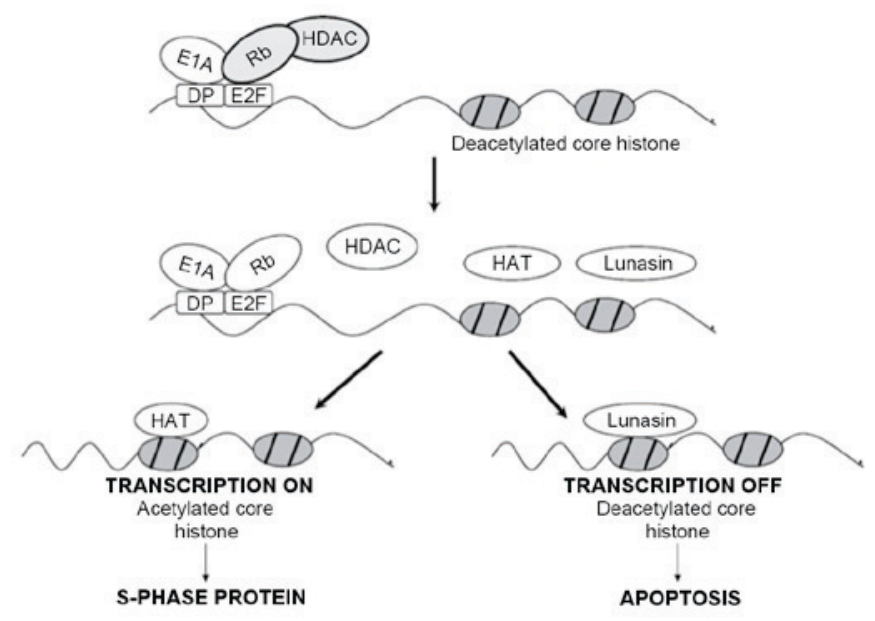

Figure 2. E1A-Rb-HDAC model describing the inhibition of E1A-induced cell transformation in the presence of lunasin. Figure previously published in (51). E1A, early region $1 \mathrm{~A}$; Rb, retinoblastoma; HDAC, histone deacetylase; DP, dimerization partner; E2F, transcription factor E2F; HAT, histone acetyltransferase.

and it was demonstrated that lunasin exhibits a limited number of side effects compared with other drugs $(32,53)$.

Previous studies have demonstrated that chronic inflammation and oxygen free radicals are involved in degenerative diseases, inflammatory diseases, cardiovascular diseases and cancer $(54,55)$. Therefore, anti-inflammation or antioxidation may be an important intervention strategy for the prevention and treatment of cancer (56). Lunasin was demonstrated to exhibit certain anti-inflammatory effects. i) Lunasin was able to inhibit the production of interleukin 6 (IL-6), tumor necrosis factor $\alpha$ and prostaglandin $\mathrm{E}_{2}\left(\mathrm{PGE}_{2}\right)$ in lipopolysaccharide (LPS)-induced RAW264.7 cells (57), and suppress the generation of IL- 6 and IL-8, as well as suppress the secretion of matrix metalloproteinases in cultured rheumatoid arthritis synovial fibroblasts through the inhibition of nuclear factor $\mathrm{kB}$ activity (58). ii) Lunasin was also able to induce the inducible nitric oxide synthase/nitric oxide signaling pathway through adjustment of the cyclo-oxygenase $2 / \mathrm{PGE}_{2}$ ratio (59). iii) Lunasin was able to perform its antioxidant function through decreasing LPS-induced reactive oxygen species production (57), and prevent the oxidative damage of DNA by inhibiting the generation of hydroxyl free radicals (60). The anti-inflammatory and antioxidant functions of lunasin improve its potential use in the prevention and treatment of cancer.

Synergistic prevention and treatment of cancer using lunasin with other anti-cancer compounds. In order to achieve a synergistic effect on the prevention and treatment of cancer, the combinatorial application of two or more drugs is a commonly used strategy, which is able to increase the treatment efficacy and decrease the toxicity of the drugs (6). In the past two decades, various studies have demonstrated that aspirin exhibits clear inhibitory activity in chemical-induced carcinogenesis $(61,62)$. However, the side effects of aspirin, including peptic ulcers, mucosal injury and bleeding, are evident (63). In order to use aspirin for its anti-carcinogenic effects and reduce its side effects, a number of studies have 
been conducted to explore the possibility of combinatorial application of aspirin and lunasin $(37,39)$. As demonstrated by previous studies, the combinatorial application of aspirin and lunasin does not result in any safety issues $(37,39)$. In addition, the combinatorial application of aspirin and lunasin resulted in a synergistic effect on the inhibition of proliferation and induction of apoptosis in human breast cancer MDA-MB-231 cells (39). This effect is primarily due to the down-regulation of $\mathrm{PKB} \alpha$, proto-oncogene Fos and AP-1 signaling genes through modulation of the genes that code for $G_{1}$ and $S$ phase regulatory proteins, thereby achieving a marked decrease in the side effects of aspirin (39). Furthermore, similar results have been observed in DMAB-induced fibroblast tumors in mice (37). However, the potential advantage of increasing anti-cancer activity and decreasing the side effects requires further study in animals and humans, and the underlying molecular mechanisms for the synergistic effects of lunasin and aspirin on the prevention and treatment of cancer require further clarification. Such studies may be of benefit for the development of a novel combinatorial treatment of cancer.

\section{Conclusions}

Numerous studies have demonstrated that lunasin may serve a role in the prevention and treatment of various types of cancer. However, the underlying molecular mechanisms of the prevention and treatment of different types of cancer or cancer stages, and the enhanced bioavailability of lunasin during oral administration remain unclear, and require further investigation or clarification using genomic or proteomic methods. In addition, the optimal fragment length, secondary or tertiary structure, or specific spatial orientation of lunasin in the prevention and treatment of cancer requires clarification. Furthermore, lunasin primarily exists in soybean, which is a principal agricultural product in China. Due to the potential activity in the prevention and treatment of cancer, and the prevention of cardiovascular diseases and inflammatory diseases, polypeptides, including lunasin from soybean, require investigation for the development of medical or healthcare products.

\section{Acknowledgements}

The present review was supported by the Natural Science Foundation of Hubei Province (grant no. 2014CFB613 received by X.C.), the Chutian Scholar Program, Hubei Superior Discipline Group of Physical Education and Health Promotion, and Outstanding Youth Scientific and Technological Innovation Team (grant no. T201624 received by N.C.) from Hubei Provincial Department of Education, and the National Natural Science Foundation of China (grant no. 31171738$)$.

\section{References}

1. Razzaghi H, Quesnel-Crooks S, Sherman R, Joseph R, Kohler B, Andall-Brereton G, Ivey MA, Edwards BK, Mery L, Gawryszewski V and Saraiya M: Leading causes of cancer mortality-Caribbean region, 2003-2013. MMWR Morb Mortal Wkly Rep 65: 1395-1400, 2016.

2. Manson MM: Cancer prevention-the potential for diet to modulate molecular signalling. Trends Mol Med 9: 11-18, 2003.
3. Zhang SF, Wang XL, Yang XQ and Chen N: Autophagy-associated targeting pathways of natural products during cancer treatment. Asian Pac J Cancer Prev 15: 10557-10563, 2014.

4. Kou X, Kirberger M, Yang Y and Chen N: Natural products for cancer prevention associated with Nrf2-ARE pathway. Food Science and Human Wellness 2: 22-28, 2013.

5. Ferlay J, Soerjomataram I, Dikshit R, Eser S, Mathers C, Rebelo M, Parkin DM, Forman D and Bray F: Cancer incidence and mortality worldwide: Sources, methods and major patterns in GLOBOCAN 2012. Int J Cancer 136: E359-E386, 2015.

6. de Kok TM, van Breda SG and Manson MM: Mechanisms of combined action of different chemopreventive dietary compounds: A review. Eur J Nutr 47 (Suppl 2): S51-S59, 2008.

7. Chen D and Dou QP: Tea polyphenols and their roles in cancer prevention and chemotherapy. Int J Mol Sci 9: 1196-1206, 2008.

8. Yang CS, Ju J, Lu G, Xiao H, Hao X, Sang S and Lambert JD: Cancer prevention by tea and tea polyphenols. Asia Pac J Clin Nutr 17 (Suppl 1): S245-S248, 2008.

9. Fimognari C, Lenzi M and Hrelia P: Chemoprevention of cancer by isothiocyanates and anthocyanins: Mechanisms of action and structure-activity relationship. Curr Med Chem 15: 440-447, 2008.

10. Udenigwe CC and Aluko RE: Food protein-derived bioactive peptides: Production, processing, and potential health benefits. J Food Sci 77: R11-R14, 2012.

11. Jia S, Du Z, Jiang H,Huang X, Chen Z and Chen N: Daintain/AIF-1 accelerates the activation of insulin-like growth factor-1 receptor signaling pathway in HepG2 cells. Oncol Rep 34: 511-517, 2015.

12. Bhutia SK and Maiti TK: Targeting tumors with peptides from natural sources. Trends Biotechnol 26: 210-217, 2008.

13. de Mejia EG and Dia VP: The role of nutraceutical proteins and peptides in apoptosis, angiogenesis and metastasis of cancer cells. Cancer Metastasis Rev 29: 511-528, 2010.

14. Liu J, Jia SH, Kirberger M and Chen N: Lunasin as a promising health-beneficial peptide. Eur Rev Med Pharmacol Sci 18: 2070-2075, 2014.

15. McCue P and Shetty K: Health benefits of soy isoflavonoids and strategies for enhancement: A review. Crit Rev Food Sci Nutr 44: 361-367, 2004.

16. Woo HD, Park S, Oh K, Kim HJ, Shin HR, Moon HK and Kim J: Diet and cancer risk in the Korean population: A meta- analysis. Asian Pac J Cancer Prev 15: 8509-8519, 2014.

17. Chi F, Wu R, Zeng YC, Xing R, Liu Y and Xu ZG: Post-diagnosis soy food intake and breast cancer survival: A meta-analysis of cohort studies. Asian Pac J Cancer Prev 14: 2407-2412, 2013.

18. Lee MM, Gomez SL, Chang JS, Wey M, Wang RT and Hsing AW: Soy and isoflavone consumption in relation to prostate cancer risk in China. Cancer Epidemiol Biomarkers Prev 12: 665-668, 2003.

19. Yamamoto S, Sobue T, Kobayashi M, Sasaki S and Tsugane S; Japan Public Health Center-Based Prospective Study on Cancer Cardiovascular Diseases G: Soy, isoflavones, and breast cancer risk in Japan. J Natl Cancer Inst 95: 906-913, 2003.

20. Messina $\mathrm{M}$ and Barnes S: The role of soy products in reducing risk of cancer. J Natl Cancer Inst 83: 541-546, 1991.

21. Toyomura K and Kono S: Soybeans, soy foods, isoflavones and risk of colorectal cancer: A review of experimental and dpidemiological data. Asian Pac J Cancer Prev 3: 125-132, 2002.

22. Clemente A and Arques Mdel C: Bowman-Birk inhibitors from legumes as colorectal chemopreventive agents. World J Gastroenterol 20: 10305-10315, 2014.

23. Galvez AF and de Lumen BO: A soybean cDNA encoding a chromatin-binding peptide inhibits mitosis of mammalian cells. Nat Biotechnol 17: 495-500, 1999.

24. Jeong HJ, Jeong JB, Kim DS, Park JH, Lee JB, Kweon DH, Chung GY, Seo EW and de Lumen BO: The cancer preventive peptide lunasin from wheat inhibits core histone acetylation. Cancer Lett 255: 42-48, 2007.

25. Jeong HJ, Lee JR, Jeong JB, Park JH, Cheong YK and de Lumen BO: The cancer preventive seed peptide lunasin from rye is bioavailable and bioactive. Nutr Cancer 61: 680-686, 2009.

26. Gonzalez de Mejia E, Vásconez M, de Lumen BO and Nelson R: Lunasin concentration in different soybean genotypes, commercial soy protein, and isoflavone products. J Agric Food Chem 52: 5882-5887, 2004.

27. Jeong HJ, Lam Y and de Lumen BO: Barley lunasin suppresses ras-induced colony formation and inhibits core histone acetylation in mammalian cells. J Agric Food Chem 50: 5903-5908, 2002 . 
28. Jeong HJ, Park JH, Lam Y and de Lumen BO: Characterization of lunasin isolated from soybean. J Agric Food Chem 51: 7901-7906, 2003.

29. Rizzello CG, Nionelli L, Coda R and Gobbetti M: Synthesis of the cancer preventive peptide lunasin by lactic acid bacteria during sourdough fermention. Nutr Cancer 64: 111-120, 2012.

30. Guijarro-Díez M, García MC, Crego AL and Marina ML: Off-line two dimensional isoelectrofocusing-liquid chromatography/mass spectrometry (time of flight) for the determination of the bioactive peptide lunasin. J Chromatogr A 1371: 117-124, 2014.

31. Hsieh CC, Hernándz-Ledesma B, Jeong HJ, Park JH and de Lumen BO: Complementary roles in cancer prevention: Protease inhibitor makes the cancer preventive peptide lunasin bioavailable. PLoS One 5: e8890, 2010.

32. Jeong JB, Jeong HJ, Park JH, Lee SH, Lee JR, Lee HK, Chung GY, Choi JD and de Lumen BO: Cancer-preventive peptide lunasin from Solanum nigrum L. inhibits acetylation of core histones $\mathrm{H} 3$ and $\mathrm{H} 4$ and phosphorylation of retinoblastoma protein (Rb). J Agric Food Chem 55: 10707-10713, 2007.

33. Dia VP, Torres S, De Lumen BO, Erdman JW Jr and De Mejia EG: Presence of lunasin in plasma of men after soy protein consumption. J Agric Food Chem 57: 1260-1266, 2009.

34. Park JH, Jeong HJ and Lumen BO: In vitro digestibility of the cancer-preventive soy peptides lunasin and BBI. J Agric Food Chem 55: 10703-10706, 2007.

35. Jiang Q, Pan Y, Cheng Y, Li H, Liu D and Li H: Lunasin suppresses the migration and invasion of breast cancer cells by inhibiting matrix metalloproteinase-2/-9 via the FAK/Akt/ERK and NF-kB signaling pathways. Oncol Rep 36: 253-262, 2016.

36. Hsieh CC, Hernandez-Ledesma B and de Lumen BO Soybean peptide lunasin suppresses in vitro and in vivo 7,12-dimethylbenz[a]anthracene-induced tumorigenesis. J Food Sci 75: H311-H316, 2010.

37. Hsieh CC, Hernandez-Ledesma B and de Lumen BO Lunasin-aspirin combination against NIH/3T3 cells transformation induced by chemical carcinogens. Plant Foods Hum Nutr 66 : 107-113, 2011.

38. Hernandez-Ledesma B,Hsieh CC and de Lumen BO: Relationship between lunasin's sequence and its inhibitory activity of histones H3 and H4 acetylation. Mol Nutr Food Res 55: 989-998, 2011.

39. Hsieh CC, Hernandez-Ledesma B and de Lumen BO: Lunasin, a novel seed peptide, sensitizes human breast cancer MDA-MB-231 cells to aspirin-arrested cell cycle and induced apoptosis. Chem Biol Interact 186: 127-134, 2010.

40. Wang W, Bringe NA, Berhow MA and Gonzalez de Mejia E: beta-Conglycinins among sources of bioactives in hydrolysates of different soybean varieties that inhibit leukemia cells in vitro. J Agric Food Chem 56: 4012-4020, 2008.

41. de Mejia EG, Wang W and Dia VP: Lunasin, with an arginine-glycine-aspartic acid motif, causes apoptosis to L1210 leukemia cells by activation of caspase-3. Mol Nutr Food Res 54 406-414, 2010

42. Galvez AF, Chen N, Macasieb J and de Lumen BO: Chemopreventive property of a soybean peptide (lunasin) that binds to deacetylated histones and inhibits acetylation. Cancer Res 61: 7473-7478, 2001.

43. Hsieh EA, Chai CM, de Lumen BO, Neese RA and Hellerstein MK: Dynamics of keratinocytes in vivo using $\mathrm{HO}$ labeling: A sensitive marker of epidermal proliferation state. J Invest Dermatol 123: 530-536, 2004.

44. Dwarakanath BS, Verma A, Bhatt AN, Parmar VS and Raj HG: Targeting protein acetylation for improving cancer therapy. Indian J Med Res 128: 13-21, 2008.
45. Dalvai $\mathrm{M}$ and Bystricky K: The role of histone modifications and variants in regulating gene expression in breast cancer. J Mammary Gland Biol Neoplasia 15: 19-33, 2010.

46. Strahl BD and Allis CD: The language of covalent histone modifications. Nature 403: 41-45, 2000.

47. Muraoka M, Konishi M, Kikuchi-Yanoshita R, Tanaka K, Shitara N, Chong JM, Iwama T and Miyaki M: p300 gene alterations in colorectal and gastric carcinomas. Oncogene 12: $1565-1569,1996$

48. Gayther SA, Batley SJ, Linger L, Bannister A, Thorpe K, Chin SF, Daigo Y, Russell P, Wilson A, Sowter HM, et al: Mutations truncating the EP300 acetylase in human cancers. Nat Genet 24: 300-303, 2000

49. Ionov Y, Matsui S and Cowell JK: A role for p300/CREB binding protein genes in promoting cancer progression in colon cancer cell lines with microsatellite instability. Proc Natl Acad Sci USA 101: 1273-1278, 2004.

50. Jeong HJ, Jeong JB, Kim DS and de Lumen BO: Inhibition of core histone acetylation by the cancer preventive peptide lunasin. J Agric Food Chem 55: 632-637, 2007.

51. Hernandez-Ledesma B and de Lumen BO: Lunasin: A novel cancer preventive seed Peptide. Perspect Medicin Chem 2: 75-80, 2008.

52. Frolov MV and Dyson NJ: Molecular mechanisms of E2F-dependent activation and pRB-mediated repression. J Cell Sci 117: 2173-2181, 2004

53. de Lumen BO: Lunasin: A novel cancer preventive seed peptide that modifies chromatin. J AOAC Int 91: 932-935, 2008.

54. Kuper H, Adami HO and Trichopoulos D: Infections as a major preventable cause of human cancer. J Intern Med 248: 171-183, 2000.

55. Allavena P, Garlanda C, Borrello MG, Sica A and Mantovani A: Pathways connecting inflammation and cancer. Curr Opin Genet Dev 18: 3-10, 2008.

56. Villegas I, Sanchez-Fidalgo S and Alarcón de la Lastra C: New mechanisms and therapeutic potential of curcumin for colorectal cancer. Mol Nutr Food Res 52: 1040-1061, 2008.

57. Hernandez-Ledesma B, Hsieh CC and de Lumen BO: Antioxidant and anti-inflammatory properties of cancer preventive peptide lunasin in RAW 264.7 macrophages. Biochem Biophys Res Commun 390: 803-808, 2009.

58. Jia S, Zhang S, Yuan H and Chen N: Lunasin inhibits cell proliferation via apoptosis and reduces the production of proinflammatory cytokines in cultured rheumatoid arthritis synovial fibroblasts. Biomed Res Int 2015: 346839, 2015.

59. Wang W, Dia VP, Vasconez M, de Mejia EG and Nelson RL: Analysis of soybean protein-derived peptides and the effect of cultivar, environmental conditions, and processing on lunasin concentration in soybean and soy products. J AOAC Int 91: 936-946, 2008.

60. Jeong JB, De Lumen BO and Jeong HJ: Lunasin peptide purified from Solanum nigrum L. protects DNA from oxidative damage by suppressing the generation of hydroxyl radical via blocking fenton reaction. Cancer Lett 293: 58-64, 2010.

61. Jacobo-Herrera NJ, Pérez-Plasencia C, Camacho-Zavala E González GF, Urrutia EL, García-Castillo V and Zentella-Dehesa A: Clinical evidence of the relationship between aspirin and breast cancer (review). Oncol Rep 32: 451, 2014.

62. Giovannucci E and Chan AT: Role of vitamin and mineral supplementation and aspirin use in cancer survivors. J Clin Oncol 28: 4081-4085, 2010

63. Laine L: Review article: Gastrointestinal bleeding with low-dose aspirin-what's the risk? Aliment Pharmacol Ther 24: 897-908, 2006. 